\title{
Thermal noise limitations on micromechanical experiments
}

\begin{abstract}
Thermal motions of microscopic probes limit the possibilities of experiments that are designed to resolve single-macromolecule dynamics in aqueous conditions. We investigate theoretical strategies for maximizing signal-to-noise ratios or resolution in typical situations, illustrating our discussion with examples from optical tweezers and atomic force microscopy experiments. A central result is that the viscous drag on a micromechanical probe is more important than the compliance of the probe. Within limits, increased stiffness of an AFM cantilever or of an optical trap does not increase resolution, and decreased stiffness does not provide the possibility of less invasive measurements.
\end{abstract}

Key words Thermal noise Optical tweezers $\cdot$ Atomic force microscopy $\cdot$ Single molecules $\cdot$ Micromechanics

\section{Introduction}

Intramolecular motions, central to biological function, have been largely inaccessible to static methods, such as electron microscopy or X-ray crystallography. Dynamics have been observed with a multitude of conventional spectroscopy methods, but these average over a macroscopic sample, making it impossible to resolve details of, for example, the motion of motor proteins or the folding and unfolding of proteins. Recently, micromechanical techniques have begun to explore the territory of small scale dynamics. Several experiments are now capable of studying the dynamics of individual biological macromolecules.

Optical tweezers have been used quantitatively to exert or measure small forces and to measure small displacements of moving objects, with sufficient resolution to study

F. Gittes · C. F. Schmidt $(\bowtie)$

Department of Physics, and Biophysics Research Division,

University of Michigan,

930 N. University,

Ann Arbor, MI 48109-1055, USA individual biological macromolecules, for example DNA and proteins (Svoboda et al. 1993; Perkins et al. 1994; Svoboda and Block 1994 b; Molloy et al. 1995; Yin et al. 1995; Coppin et al. 1996; Simmons et al. 1996; Smith et al. 1996; Kellermayer et al. 1997; Stout and Webb 1997; Tskhovrebova et al. 1997; Wang et al. 1997). Optical trapping of particles uses the momentum transfer from light scattered or diffracted by an object (Ashkin et al. 1986; Ashkin 1992; Ashkin 1997). Three-dimensional trapping of particles can be achieved at the focus of a laser beam if a strong enough gradient of intensity can be established in all directions. Typical forces are on the order of tens of piconewtons (pN) (Svoboda and Block 1994a).

Atomic force microscopy (AFM) (Moy et al. 1994; Thomson et al. 1996; Rief et al. 1997), although initially developed to image surfaces (Rugar and Hansma 1990), glass microneedles (VanBuren et al. 1994; Meyhofer and Howard 1995; Cluzel et al. 1996; Ishijima et al. 1996), magnetic beads (Smith et al. 1992; Strick et al. 1996) and single molecule fluorescence microscopy (Funatsu et al. 1995; Sase et al. 1995) have also been used to study individual molecules.

In single-molecule experiments, evading noise in various forms becomes of foremost importance. Noise appears in electronic components, but is also unavoidably present as the Brownian motion of the observed object and of the probe that is interacting with the object, which are typically immersed in room-temperature aqueous solutions. Thermal noise sources set fundamental limits to micromechanical force and position measurements. Intuition is often misleading in dealing with thermal fluctuations on $\mathrm{nm}$ length scales. One possible misconception is that, in attempts to image soft molecules with AFM (for a review see (Shao et al. 1996)), softer cantilevers allow less invasive imaging. It has similarly been argued that a stiffer optical trap decreases thermal noise (Visscher et al. 1996), which does, however, not necessarily mean that the signalto-noise ratio is improved. Here we will discuss noise issues with an eye on optical tweezers experiments and AFM, but the basic results of the discussion apply to other micromechanical experiments as well. 
Common to many of these techniques is non-imaging detection with fast photodiodes. Intense illumination can be used to track the motion of objects with $\AA$-accuracy (Bobroff 1986; Denk and Webb 1990). Using that fact, the motion of an AFM cantilever is usually detected by a laser beam reflected off the back of the cantilever onto a split photodiode (Meyer and Amer 1988; Alexander et al. 1989). With optical tweezers the trapping laser beam itself can be used for position detection (Svoboda and Block 1994a; Smith et al. 1996).

\section{Spectral data analysis}

Experiments that directly monitor microscopic dynamics usually produce time-domain data, $x(t)$, such as a series of voltage measurements corresponding to the varying light intensity detected with a photodiode. The time series is obviously relevant for examining single events. Instrumental and thermal noise, on the other hand, is best characterized in the frequency domain by its power spectrum or power spectral density (PSD), here denoted by $S_{x}(f)$. $S_{x}(f)$ is defined as the Fourier transform of the autocorrelation function of the time series. It is more conveniently estimated from the squared magnitude of the Fourier transform of data sets: $S_{x}(f)=n \cdot \overline{|\tilde{x}(f)|^{2}}$, where $\tilde{x}(f)$ is the Fourier transform of $x(t)$, and $n$ normalizes $S_{x}(f)$ so that its frequency integral equals the variance of the data. The finite length of the data set and the sampling time affect this estimate of $S_{x}(f)$; such elementary aspects of power spectrum calculation can be found elsewhere (Press et al. 1992; Gittes and Schmidt 1997).

\section{Brownian motion of a harmonically bound particle}

For systems such as optically trapped particles in solution, or a microscopic AFM tip, the simplest theory of Brownian motion relies on the Langevin equation, which models fluctuations as a response, via the hydrodynamic drag coefficient of the object, to a microscopic random thermal force. This formalism is justified by the fluctuation-dissipation theorem (Reif 1965; Landau et al. 1980), which generally relates linear "dissipation" coefficients to the properties of thermal fluctuations. In an optical trap, the motion of a micron-scale trapped particle takes place at small Reynolds number, so that viscous drag is completely dominant over inertial forces (Happel and Brenner 1983). The Langevin equation for such as Brownian harmonic oscillator is

$\gamma \dot{x}+\kappa x=F(t)$

with hydrodynamic drag coefficient $\gamma$, particle position $x$ and velocity $\dot{x}$. The spring stiffness of the harmonic potential is $\kappa$, and the random force is $F(t)$, with an average value of 0 , and a constant power spectral density $S_{F}(f)=n \cdot \overline{|\tilde{F}(f)|^{2}}=$ constant (white noise). This ap- proximation is valid at frequencies sufficiently lower than those of the rapid variations in solvent forces $\left(10^{14} \mathrm{~Hz}\right)$. The Nyquist theorem is a special case of the fluctuationdissipation theorem, originally describing thermal voltage fluctuations in electrical circuits, but which directly translates to this mechanical system (Reif 1965; Landau et al. 1980) (sec. 15.5, 15.8, 15.17). The Nyquist formula expresses the thermal white-noise force magnitude in terms of $\gamma$,

$S_{F}(f)=4 \gamma k_{B} T$,

where $k_{B}$ is Boltzmann's constant and $T$ is absolute temperature. If the drag coefficient becomes complex-valued (see below), $\gamma$ in the Nyquist formula is replaced by its real part. By Fourier transforming both sides of Eq. (1), taking their average square magnitude, and using Eq. (2), one finds that the power spectrum of the position signal is

$S_{x}(f)=\frac{k_{B} T}{\gamma \pi^{2}\left(f_{c}^{2}+f^{2}\right)}$.

where we define the characteristic frequency $f_{c}=\kappa / 2 \pi \gamma$. For frequencies $f \ll f_{c}$, the power spectrum is approximately constant, $S_{x}(f) \approx S_{0}=4 \gamma k_{B} T / \kappa^{2}$, which reflects the confinement of the particle. At higher frequencies, $f \gg f_{c}, S_{x}(f)$ falls off like $1 / f^{2}$, characteristic of free diffusion - over short times the particle does not "feel" the confinement.

The motion of an AFM tip immersed in fluid can also be described by some overall drag coefficient, $\gamma$. The Nyquist formula (Eq. (2)) will hold, and thermal forces will depend only upon the drag coefficient. However, the tip may be underdamped, even in a liquid. In the presence of an inertial resonance, the spectrum of noise fluctuations is no longer a simple Lorentzian, and a peak appears in the PSD. A typical resonant frequency for an AFM cantilevers is $\sim 10 \mathrm{kHz}$ with the tip submerged in fluid, with a Q-value of less than 10 (Roters and Johannsmann 1996). A more subtle point is that, due to a possibly large region of contact between the cantilever and the fluid, inertial shear motion in the fluid could cause $\gamma$ to be both complex (i.e. exhibiting a phase lag) and weakly frequency-dependent over some frequencies of interest, even at low Reynolds number (Landau and Lifshitz 1959). However, such an effect has not been detected to our knowledge, and might be insignificant. We will here assume $\gamma$ to be real.

\section{Hydrodynamic drag}

The viscous drag coefficient $\gamma$ of an object can in some cases be calculated by solving hydrodynamic equations of motion. One such solution is the Stokes drag on a small sphere, far from any surface (Reif 1965): $\gamma=6 \pi \eta a$, where $\eta$ is the dynamic viscosity of the solvent and $a$ is the radius of the sphere. Other unbounded-fluid drag coefficients, which we denote as $\gamma_{\infty}$, are available for objects of various shapes (Happel and Brenner 1983). When an ob- 
ject is located close to a surface (at height $h$ ) as in a typical AFM application, the drag coefficient $\gamma(h)$ that must be used in Eq. (2) is different from $\gamma_{\infty}$. One also must use $\gamma(h)$, not $\gamma_{\infty}$, when flow is induced above a surface and the object is held stationary at height $h$. For a sphere at a distance larger than its radius from a surface, the first-order correction to the Stokes equation is the Lorentz formula (Happel and Brenner 1983):

$$
\gamma=6 \pi \eta a\left(1+\frac{9}{16} \frac{a}{h}\right)
$$

which describes motion parallel to the wall with the sphere center at a height $h$. For motion normal to the wall, the factor $9 / 16$ is replaced by $9 / 8$. As the gap $h-a$ vanishes, the drag coefficient becomes infinite (Goldman et al. 1966):

$$
\begin{aligned}
& \gamma(h) \cong \gamma_{\infty} \times a /(h-a) \quad \text { (perpendicular motion) } \\
& \gamma(h) \cong \gamma_{\infty} \times \log [a /(h-a)] \quad \text { (parallel motion) }
\end{aligned}
$$

with $\gamma_{\infty}=6 \pi \eta a$. Equations (5) assume a very low Reynolds number. They are frequency-independent, up to rather high frequencies (about $1 \mathrm{MHz}$, for a micron-diameter bead), where inertial shear effects become significant (Landau and Lifshitz 1959).

\section{Thermal noise in typical experiments}

Micromechanical experiments measure forces and displacements produced by a molecule or surface of interest (the "target" object). This is typically done by monitoring the position $x_{p}(t)$ of an elastically suspended probe as it interacts with the target object (Fig. 1). This probe could be an optically trapped particle (Fig. 1 A), in which case the displacement $\Delta x(t)=x_{p}(t)-x_{0}(t)$ of the probe from the trap center $x_{0}(t)$ is typically measured. The probe could also be an AFM tip scanned horizontally across a surface, or moved vertically to interact with a fixed macromolecule (Fig. 1 B). In the latter case the probe displacement $\Delta x$ from its resting position is inferred from the distortion of the elastic cantilever. We will generally call $\Delta x(t)$ the "probe strain". The suspension force is inferred from the stiffness $K_{p}$ of the elastic element as

$F(t)=K_{p} \Delta x(t)$.

Position detection can be used to construct a feedback loop, to move the zero-force position of the probe relative to the sample (in AFMs, it is often the sample that actually moves).

We discuss two prototypical feedback experiments - although other experiments (e.g. without feedback) may be intermediate cases. Different types of detectors are necessary for these two types of experiments. Using piezoelectric actuators (in AFM) or acousto-optic or electro-optic modulators (with optical tweezers) to move the anchor point of the probe assembly, ideal feedback can be approximated quite well.
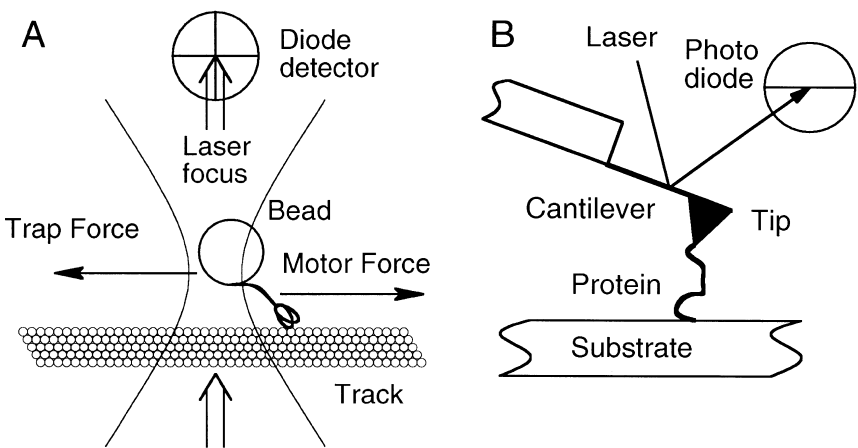

Fig. 1 A, B Two examples of single molecule micromechanical experiments. A Optical tweezers: a laser focus traps a micron sized bead, with a motor protein attached to it. The motor protein moves along a track protein. The force on the motor and its displacement from the trap center can be measured with a photo diode. B AFM: a flexible cantilever carries a microscopically sharp tip, here shown interacting with a macromolecule fixed to a substrate. Tip deflection is monitored with a laser and a photodiode

\section{Position-clamp experiments}

Position clamp experiments measure a time-varying force $F_{\text {sig }}(t)$, exerted on a probe held stationary by using feedbacl on the anchor position $x_{0}(t)$ (Fig. 2A). In an AFM, this might be used to study unfolding of proteins (Rief et al. 1997) and, in an optical trap, to measure the force production of a molecular motors under a stationary load (Molloy et al. 1995; Simmons et al. 1996; Wang et al. 1997). As a changing force begins to displace the probe, feedback changes the probe strain $\Delta x(t)=x_{p}-x_{0}(t)$ to keep the probe position $x_{p}$ constant. The time-dependent force is found from $\Delta x(t)$ :

$F_{\text {tot }}(t)=K_{p} \Delta x(t)$

With perfect feedback control, the fundamental limitation in measuring the force due to the target objects comes from white-noise thermal force that also acts on the probe. The power spectrum of thermal force is given by Eq. (2), where $\gamma$ is the frictional drag coefficient of the probe in the fluid.

Because the thermal noise power extends to very high frequencies, low-pass filtering the strain signal $\Delta x(t)$ will increase the signal-to-noise ratio (the cut-off frequency $f_{s}$ must of course lie above frequencies of interest in the signal). The remaining uncertainty $\Delta F(t)=\left(F_{t o t}(t)-F_{\text {sig }}(t)\right)_{r m s}$ is the integrated noise power below $f_{s}$,

$$
\Delta F_{r m s}=\sqrt{\Delta F^{2}}=\sqrt{4 \gamma k_{B} T f_{s}} .
$$

The force uncertainty is minimized by either: (i) reducing the drag $\gamma$ on the probe, or (ii) slowing down the force signal to be measured and making $f_{s}$ as low as possible - by scanning slowly with an AFM, for example. In the case of a bead of diameter $1 \mu \mathrm{m}$, optically trapped (in water far from a surface) the drag coefficient is about $\gamma=8 \times 10^{-9} \mathrm{~kg} / \mathrm{s}$. Assuming a reasonable bandwidth of $1 \mathrm{kHz}$, this implies a force uncertainty of $\Delta F_{r m s} \sim 0.4 \mathrm{pN}$. For an AFM tip, the level of noise may be much larger due 


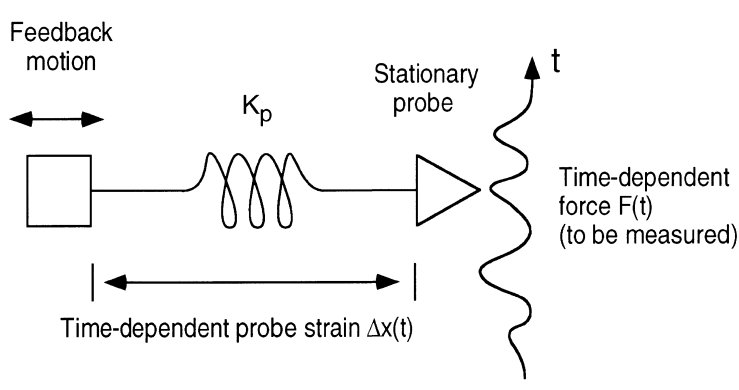

B FORCE-CLAMP EXPERIMENT

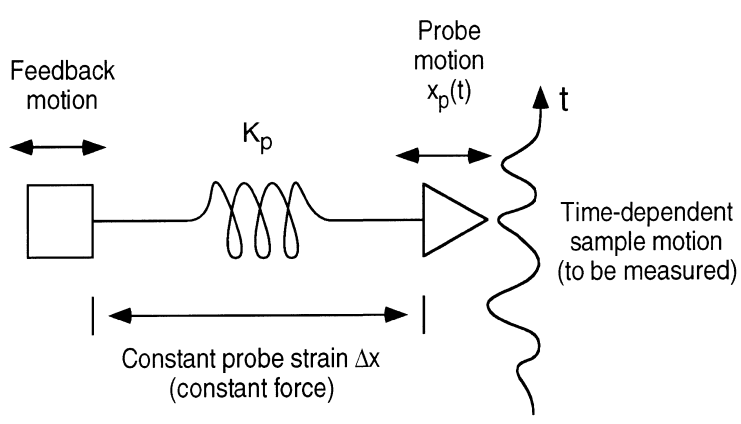

Fig. 2 A, B Two schematic prototypical micromechanical experiments with feedback. In an optical trap, the probe is a trapped dielectric particle, the anchor point (square) represents the position of the center of the trap, which is controlled through feedback, and $K_{p}$ represents the trap stiffness. In an AFM, the probe is the scanning tip, the anchor point is the base of the cantilever, (controlled via feedback) and $K_{p}$ is the cantilever stiffness. The probe interacts with the sample through a force that may change with (lateral) position and time. A Position-clamp experiment. Absolute probe position is monitored and the anchor point is moved to keep the probe stationary. From the changing probe strain, the changing force on the probe is known. B Force-clamp experiment. The anchor point is now moved to keep a constant probe strain, and thus a constant force on the probe. The anchor motion then shows the constant-force motion. However, this probe response is low-pass filtered by the dynamic response characteristics of the probe

to the larger drag of the tip, especially close to a surface. This situation implies a trade-off between temporal and force resolution and this may be a serious problem, for example, in measurements of the dynamics of protein unfolding (Rief et al. 1997). Static forces can in principle be measured to arbitrary precision, with very small $f_{s}$ and very long measurement times, but, in practice, drift in the apparatus becomes limiting. Equation (8) shows that the stiffness of the elastic probe suspension if not relevant in principle. In practice, electronic noise in the strain detector can limit how small a strain can be detected, in which case a softer probe allows measurement of a smaller force change and of a smaller absolute force. On the other hand, electronic noise and laser noise can be controlled, so that detector noise is usually not the limiting factor.

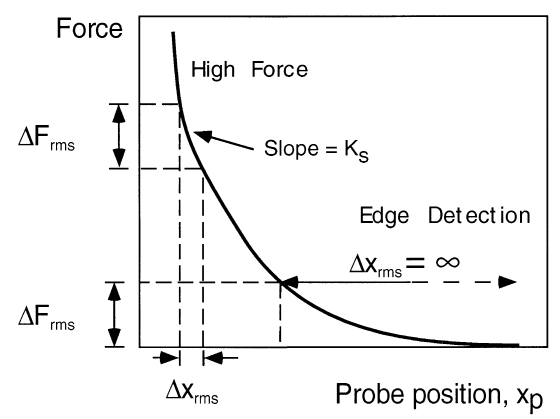

Fig. 3 Force-clamp experiments at high force and at low force (edge detection). The solid curve is an instantaneous force profile $F\left(x_{p}\right)$ as a function of probe position, $x_{p}$. An uncertainty $\Delta F_{r m s}$ in force measurement results from thermal forces on the probe. High force: the apparatus is operating in a constant-force mode at values $F_{s e t}$ well above the force uncertainty, $F_{\text {set }} \gg \Delta F_{r m s}$. The force uncertainty translates, via the slope $K_{s}$ of $F\left(x_{p}\right)$, into an uncertainty in locating the position $x\left(F_{\text {set }}\right)$ on the force profile corresponding to the set force. Edge detection: locating the "edge" of a profile in the least invasive manner, i.e. using the smallest possible force. The smallest possible set force is $F_{s e t} \approx \Delta F_{r m s}$. If $F_{\text {set }}$ approaches $\Delta F_{r m s}$ the position uncertainty diverges to infinity

\section{Force-clamp experiments}

If the probe strain $\Delta x$ is held constant by feedback as the probe moves, the constant suspension force $F_{s e t}=K_{p} \Delta x$ is balanced by a constant force of interaction with the target (Fig. 2B). The probe position $x_{p}(t)$ is then monitored. With an AFM one might be tracing a surface or the shape of a biological macromolecule as defined by its constant-force contours. In an optical trap, one might be following the motion of a molecular motor under a constant load. We momentarily ignore direct dynamical effects of viscous drag and cantilever mass (and return to this below); thus in the absence of thermal forces the probe would always exert $e x$ actly the chosen force on the sample. In reality, the suspension force actually balances the constant sum of the force of interaction with the target and a fluctuating thermal force on the probe. The probe position $x_{p}(t)$ is then only an estimate for the true constant-load position of the target.

Two experimental goals need to be distinguished. Figure 3 illustrates the situation with a hypothetical interaction-force profile between probe and sample (for example, repulsive force increases when an AFM tip approaches a surface; alternatively the elastic linkage between a probe and a molecular motor develops tension with increasing distance). Thermal noise imposes distinct limitations in two experimental situations: (i) It limits the accuracy with which a high-force spatial response can be determined, and (ii) it sets a minimum force at which a spatial response can be obtained at all.

\section{Displacement measurements at large constant force}

Displacement response $x_{p}(t)$ to a strong force $F_{\text {set }}$ may include an (intentional) deformation of the target, such as the 
unfolding of a protein. In our context, however, it is irrelevant what mechanism produces $x_{p}(t)$. By a "large" force we simply mean that $F_{\text {set }}$ is large compared to the rootmean-square thermal force on the probe: $F_{\text {set }} \gg \Delta F_{r m s}$ (see Eq. (8)). The position uncertainty in the experiment is caused by the force uncertainty $\Delta F_{r m s}$ (Fig. 3 ). If the local stiffness of the probe-sample interaction is $K_{S}$ (i.e. the local slope of $F$ vs. $x$ in Fig. 3), then

$$
\Delta x_{r m s}=\frac{\sqrt{4 \gamma k_{B} T f_{s}}}{K_{s}} \text {. }
$$

As with the position clamp, the stiffness of the trap or the cantilever does not enter directly, but now the details of the probe-target interaction are determining the error. In measuring displacement $x_{p}(t)$ caused by molecular motor action with optical tweezers, for example, the uncertainty $\Delta x_{r m s}$ can be very small if the stiffness $K_{s}$ of the bead-motor linkage is high. Thermal noise can be further reduced, as before, by reducing the drag on the probe, or by slowing the target motion if possible.

\section{"Edge" detection}

Sometimes one wants to detect the edge of a force profile without disturbing the object. In imaging soft biomolecules with an AFM, one would, for example, like to follow the lowest possible force contour. In any real system, the interaction force between probe and sample will smoothyl approach zero at some distance (Fig. 3). Again ignoring direct dynamical effects of drag and inertia, the suspension force $F_{\text {set }}$ again balances the sum of interaction force with the target object, $F_{o b j}$, and the random thermal force $\Delta F(t)$ given by Eq. (8). The thermal noise now determines the lowest force contour that can be followed. As an example, consider a constant-force AFM probe near a repulsive surface. If a thermal fluctuation $\Delta F(t)$ pushes the probe away from the surface, the feedback will move the probe even further to decrease $F_{o b j}(t)$ to compensate for the increased thermal force. If $F_{s e t}$ is so low that $F_{s e t}+\Delta F(t)$ becomes negative, the feedback cannot compensate even by moving completely away from the surface. To locate the "edge" of a force profile, one must therefore apply at least a force $F_{s e t} \approx \Delta F_{r m s}=\sqrt{4 \gamma k_{B} T f_{s}}$.

For noise reduction, again the primary strategies are to reduce both the drag on the probe and the filter frequency $f_{s}$. Surprisingly, probe stiffness is again not a direct consideration in avoiding large forces on the sample (assuming the detector resolution not to be limiting).

\section{Displacement measurement without feedback}

AFM tips are often scanned without feedback, with the passive cantilever compliance allowing motion (these are often called "constant-height" scans because of the constant anchor height, not to be confused with our positionclamp experiments). With optical tweezers, displacements of a single active molecule against the trap compliance are often measured without using feedback. The molecule is tethered by a compliant link (stiffness $K_{s}$ ) to the probe held in the trap ( $\operatorname{stiffness} K_{p}$ ). Due to the compliant attachment, the molecular displacement, $\delta x_{m}$, will result in an attenuated probe displacement, $\delta x_{p}$ :

$\delta x_{p}=\delta x_{m} \frac{K_{s}}{K_{s}+K_{p}}$

Due to thermal forces, the position uncertainty of the probe will be (analogous to Eq. 9):

$$
\Delta\left(\delta x_{p}\right)_{r m s}=\frac{\sqrt{4 \gamma k_{B} T f_{s}}}{K_{s}+K_{p}}
$$

The signal-to-noise ratio can then be defined as:

$$
\frac{\delta x_{p}}{\Delta\left(\delta x_{p}\right)_{r m s}}=\frac{K_{s} \delta x_{m}}{\sqrt{4 \gamma k_{B} T f_{s}}}
$$

The signal-to-noise ratio is therefore independent of trap stiffness, and again dependent on filter frequency and drag coefficient. The signal-to-noise ratio also becomes larger with increasing stiffness in the molecule-to-probe connection; this has been observed (Svoboda et al. 1993).

\section{Dynamic response of the probe interacting with a sample}

Another unavoidable limitation for micromechanical experiments is the dynamic response of the probe, which is not actually a noise effect. The probe cannot respond instantaneously to the motion of the sample because of its dynamic properties (viscous drag, mass and stiffness). If these dynamic properties depend specifically on the nature of the sample-probe interaction, which is to be measured and therefore a priori unknown, then a fundamental ambiguity remains. This is true no matter how perfect the feedback is. As an example, if an AFM scan is made at too high a scan rate, the probe will not follow a compliant surface, but will simply plow through at nearly constant height. Or, if a protein changes conformation very rapidly, an attached probe may not be able to immediately follow that change.

To be specific, consider a probe suspended with finite stiffness $K_{p}$, drag $\gamma$ and mass $m$. If these were all zero, an "ideal" probe motion, $x_{p 0}(t)$, would be measured. In an actual measurement, inertia, drag and stiffness cause the actual probe motion $x_{p}(t)$ to more or less lag behind $x_{p 0}(t)$. The equation of motion for the probe contains then elastic forces, drag force and a mass term, where usually the stiffness of the probe-object interaction, $K_{s}$, is unknown:

$m \ddot{x}_{p}+\gamma \dot{x}_{p}+\left(K_{p}+K_{s}\right) x_{p}=K_{s} x_{p 0}(t)$.

We consider non-zero frequencies, so that no DC term appears. Fourier transforming Eq. (13) shows the magnitudes of the Fourier components at a given frequency $f, \tilde{x}_{p}(f)$ and $\tilde{x}_{p 0}(f)$ to be related by

$$
\left|\tilde{x}_{p}(f)\right|=\frac{K_{s}}{\left[\left(K_{p}+K_{s}-4 \pi^{2} m f^{2}\right)+4 \pi^{2} \gamma^{2} f^{2}\right]^{1 / 2}} \cdot\left|\tilde{x}_{p 0}(f)\right| .
$$


For soft samples (small $K_{s}$ ), this prefactor may contain a sample-dependent resonance near the frequency $f=\left(\left(K_{p}+K_{s}\right) / m\right)^{1 / 2}$, shifted from the resonance in solution (see above); variation in $K_{s}$ and other sample properties may in fact be deduced from changes in frequency and width of this free solution resonance (Roters and Johannsmann 1996). However, if the unperturbed signal $\tilde{x}_{p 0}(f)$ is desired, it may not be separable from the prefactor containing the unknown parameter $K_{s}$. For example, take a perfect force-clamp experiment using a massless probe, for which we can put $K_{p}=m=0$ in Eqs. (13) and (14), and the prefactor becomes $f_{p s} / \sqrt{f_{p s}^{2}+f^{2}}$. Due to probe response, the true signal $\tilde{x}_{p 0}(f)$ is both attenuated and low-pass filtered at a frequency $f_{p s}=K_{s} / 2 \pi \gamma$ that is unknown from this experiment alone.

In experiments measuring motor protein forces, the stiffness $K_{s}$ of the motor-bead linkage was found to be variable, between about 0.01 and $0.1 \mathrm{pN} / \mathrm{nm}$ (Svoboda et al. 1993; Svoboda and Block 1994b; Kuo et al. 1995; Meyhofer and Howard 1995; Coppin et al. 1996), which for $0.5 \mu \mathrm{m}$ beads implies a cutoff frequency on the order of $1 \mathrm{kHz}$. On the other hand, AFM probes against protein surfaces, which typically have elastic moduli of several GPa (Gittes et al. 1993), show effective spring constants $K_{s}$ on the order of $10^{2} \mathrm{pN} / \mathrm{nm}$, which for a low-drag probe could lead to very high cutoff frequencies.

Without feedback the situation can be analyzed along the same lines. With mechanical resonances, on the other hand, feedback can become difficult due to additional phase shifts in the loop, and practical situations may be very complex.

\section{Conclusions}

Single molecule dynamic experiments at room temperature are severely affected by thermal fluctuations in microscopic probes and in the molecules themselves. There are some universal but sometimes counterintuitive strategies to increase the signal-to-noise ratio. Low pass filtering and reducing viscous drag on the probe directly improve resolution, but probe stiffness does not play a direct role. Neither does a soft probe in general help to measure or image more gently, nor does a stiff probe help to decrease noise. For fast motions, viscous drag forces and inertial forces on the probe need to be taken into account, with the consequence that at high frequencies a true constant force experiment is not possible.

Acknowledgements We acknowledge detailed discussions with Winfried Denk, who first pointed out that force resolution is independent of probe stiffness in micromechanical measurements, and with Karel Svoboda. We acknowledge support from the National Science Foundation (grant \#BIR-9512699), the Whitaker Foundation, and the donors of the Petroleum Research Foundation, administered by the ACS.

\section{References}

Alexander S, Hellemans L, Marti O, Schneir J, Elings V, Hansma PK, Longmire M, Gurley J (1989) An atomic-resolution atomicforce microscope implemented using an optical lever. J Appl Phys 65: $164-167$

Ashkin A (1992) Forces of a single-beam gradient laser trap on a dielectric sphere in the ray optics regime. Biophys J 61:569582

Ashkin A (1997) Optical trapping and manipulation of neutral particles using lasers. Proc Natl Acad Sci USA 94:4853-4860

Ashkin A, Dziedzic JM, Bjorkholm JE, Chu S (1986) Observation of a single-beam gradient force optical trap for dielectric particles. Opt Lett 11:288-290

Bobroff N (1986) Position measurement with a resolution and noiselimited instrument. Rev Sci Instrum 57:1152-1157

Cluzel P, Lebrun A, Heller C, Lavery R, Viovy JL, Chatenay D, Caron F (1996) DNA - an Extensible Molecule. Science 271:792-794

Coppin CM, Finer JT, Spudich JA, Vale RD (1996) Detection of sub8-nm movements of kinesin by high-resolution optical-trap microscopy. Proc Natl Acad Sci USA 93: 1913-1917

Denk W, Webb WW (1990) Optical measurement of picometer displacement of transparent microscopic objects. Appl Opt 29: 2382-2391

Funatsu T, Harada Y, Tokunaga M, Saito K, Yanagida T (1995) Imaging of single fluorescent molecules and individual ATP turnovers by single myosin molecules in aqueous solution. Nature 374:555-559

Gittes F, Mickey B, Nettleton J, Howard J (1993) Flexural rigidity of microtubules and actin filament measured from thermal fluctuations in shape. J Cell Biol 120:923-934

Gittes F, Schmidt CF (1997) Signals and noise in micromechanical measurements. In: Sheetz MP (ed) Laser tweezers in cell biology. Academic Press, San Diego, CA (in press)

Goldman AJ, Cox RG, Brenner H (1996) The slow motion of two identical arbitrarily oriented spheres through a viscous fluid. Chem Eng Sci 21:1151-1170

Happel J, Brenner H (1983) Low Reynolds number hydrodynamics: with special applications to particulate media, 1st pbk. ed edn. M. Nijhoff (Distributed by Kluwer, Boston), The Hague Boston Hingham, MA, USA

Ishijima A, Kojima H, Higuchi H, Harada Y, Funatsu T, Yanagida T (1996) Multiple- and single-molecule analysis of the actomyosin motor by nanometer-piconewton manipulation with a microneedle: unitary steps and forces. Biophys J 70:383-400

Kellermayer MS, Smith SB, Granizer HL, Bustamante C (1997) Folding-unfolding transitions in single titin molecules characterized with laser tweezers. Science 276: 1112-1116

Kuo SC, Ramanathan K, Sorg B (1995) Single kinesin molecules stressed with optical tweezers. Biophys J 68:74S

Landau LD, Lifshitz EM (1959) Fluid mechanics. Pergamon Press, Addison-Wesley Pub. Co., London Reading, MA

Landau LD, Lifshitz EM, Pitaevskii LP (1980) Statistical physics. Pergamon Press, Oxford New York

Meyer G, Amer NM (1988) Novel optical approach to atomic force microscopy. Appl Phys Lett 53: 1045-1047

Meyhofer E, Howard J (1995) The force generated by a single kinesin molecule against an elastic load. Proc Natl Acad Sci USA 92:574-578

Molloy JE, Burns JE, Kendrick-Jones J, Tregear RT, White DC (1995) Movement and force produced by a single myosin head. Nature 378:209-212

Moy VT, Florin EL, Gaub HE (1994) Intermolecular forces and energies between ligands and receptors. Science 266:257-259

Perkins TT, Quake SR, Smith DE, Chu S (1994) Relaxation of a single DNA molecule observed by optical microscopy. Science 264: 822-826

Press WH, Flannery BP, Teukolsky SA, Vetterling WT (1992) Numerical Recipes in C: the art of scientific computing, 2nd edn. Cambridge University Press, Cambridge, New York 
Reif F (1965) Fundamentals of statistical and thermal physics. McGraw-Hill, New York

Rief M, Gautel M, Oesterhelt F, Fernandez JM, Gaub HE (1997) Reversible unfolding of individual titin immunoglobulin domains by AFM. Science 276: 1109-1112

Roters A, Johannsmann D (1996) Distance-dependent noise measurements in scanning force microscopy. J Phys: Condens Matter 8:7561-7577

Rugar D, Hansma P (1990) Atomic force microscopy. Physics Today 43:23-30

Sase I, Miyata H, Corrie JE, Craik JS, Kinosita K, Jr. (1995) Real time imaging of single fluorophores on moving actin with an epifuorescence microscope. Biophys J 69:323-328

Shao Z, Mou J, Czajkowsky DM, Yang J, Yuan J-Y (1996) Biological atomic force microscopy: what is achieved and what is needed. Adv Phys 45: 1-86

Simmons RM, Finer JT, Chu S, Spudich JA (1996) Quantitative measurements of force and displacement using an optical trap. Biophys J 70: 1813-1822

Smith SB, Cui YJ, Bustamante C (1996) Overstretching B-DNA the elastic response of individual double-stranded and singlestranded DNA molecules. Science 271:795-799

Smith SB, Finzi L, Bustamante C (1992) Direct mechanical measurements of the elasticity of single DNA molecules by using magnetic beads. Science 258:1122-1126

Stout AL, Webb WW (1997) Kinetics under load: the protein A immunoglobulin $\mathrm{G}$ interaction investigated using optical tweezers. Biophys J 72: A 237
Strick TR, Allemand JF, Bensimon D, Bensimon A, Croquette V (1996) The elasticity of a single supercoiled DNA molecule. Science 271:1835-1837

Svoboda K, Block SM (1994a) Biological applications of optical forces. Annu Rev Biophys Biomol Struct 23:247-285

Svoboda K, Block SM (1994b) Force and velocity measured for single kinesin molecules. Cell 77:773-784

Svoboda K, Schmidt CF, Schnapp BJ, Block SM (1993) Direct observation of kinesin stepping by optical trapping interferometry. Nature 365:721-727

Thomson NH, Fritz M, Radmacher M, Cleveland JP, Schmidt CF, Hansma PK (1996) Protein tracking and detection of protein motion using atomic force microscopy. Biophys J 70:2421-2431

Tskhovrebova L, Trinick J, Sleep JA, Simmons RM (1997) Elasticity and unfolding of single molecules of the giant muscle protein titin. Nature 387:308-312

VanBuren P, Work SS, Warshaw DM (1994) Enhanced force generation by smooth muscle myosin in vitro. Proc Natl Acad Sci USA 91:202-205

Visscher K, Gross SP, Block SM (1996) Construction of multiplebeam optical traps with nanometer-resolution position sensing. IEEE J Selected Topics in Quantum Electronics 2: 1066-1076

Wang MD, Yin H, Landick R, Gelles J, Block SM (1997) Stretching DNA with optical tweezers. Biophys J 72:1335-1346

Yin H, Wang MD, Svoboda K, Landick R, Block SM, Gelles J (1995) Transcription against an applied force. Science 270:1653-1657 